\title{
Basement membranes*
}

\author{
James M. Kramer ${ }^{\S}$, Department of Cell and Molecular Biology, \\ Northwestern University Medical School, Chicago, IL 60611 USA
}

\section{Table of Contents}

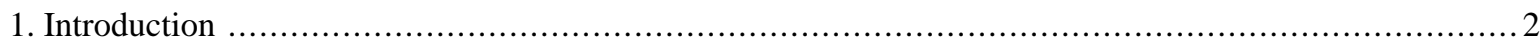

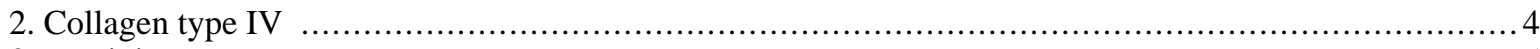

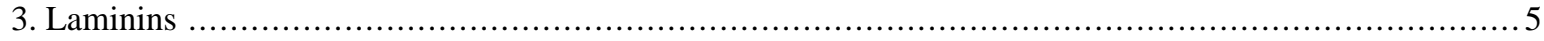

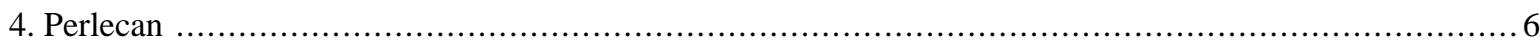

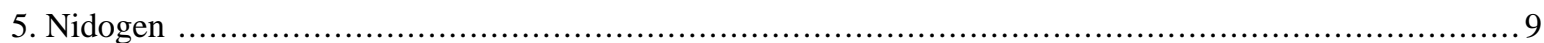

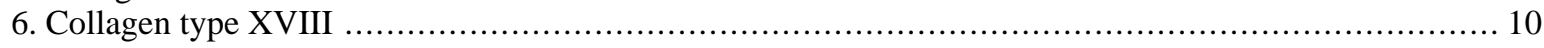

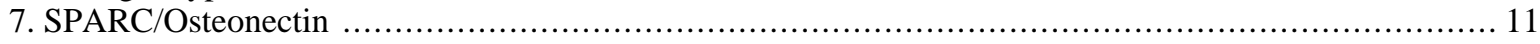

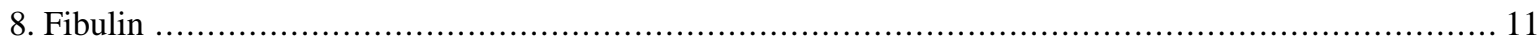

9. Hemicentin

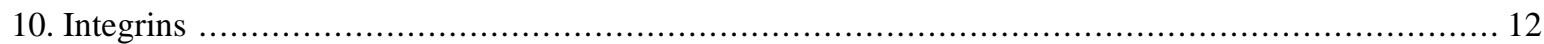

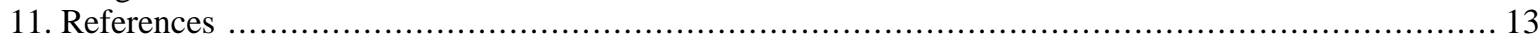

\begin{abstract}
Basement membranes are thin, specialized extracellular matrices surrounding most tissues in all metazoans. The compositions and functions of basement membranes have generally been well conserved throughout the subkingdom. Genetic analyses of basement membrane components in $C$. elegans have provided insights into their assembly and functions during development. Immuno- or GFP-tagged localization studies have shown that basement membranes on different tissues, or even sub-regions of tissues, contain different sets of proteins or alternatively spliced isoforms of them. Several components, including laminin, perlecan, type IV collagen and possibly osteonectin/SPARC, are essential for completion of embryogenesis, being necessary for tissue organization and structural integrity. In contrast, type XVIII collagen and nidogen are not required for viability but primarily influence organization of the nervous system. All of these proteins, with the exception of nidogen and the addition of fibulin, have roles of varying degree in morphogenesis of the gonad. A major family of cellular receptors for basement membrane proteins, the integrins, have also been characterized in C. elegans. As one might expect, integrins have been shown to function in many of the same processes as their potential ligands, the basement membrane components. While much remains to be explored, studies of basement membranes in $C$. elegans have been highly informative and hold great promise for improving our understanding of how these structures are assembled and how they function in development.
\end{abstract}

\footnotetext{
${ }^{*}$ Edited by Donald G. Moerman. Last revised August 31, 2005. Published September 1, 2005. This chapter should be cited as: Kramer, J.M. Basement membranes (September 1, 2005), WormBook, ed. The C. elegans Research Community, WormBook, doi/10.1895/wormbook.1.16.1, http://www.wormbook.org.

Copyright: (C) 2005 James M. Kramer. This is an open-access article distributed under the terms of the Creative Commons Attribution License, which permits unrestricted use, distribution, and reproduction in any medium, provided the original author and source are credited.

${ }^{\S}$ To whom correspondence should be addressed. E-mail: jkramer@northwestern.edu
} 


\section{Introduction}

In $C$. elegans there are two major forms of extracellular matrix, the cuticle (exoskeleton) and basement membranes (BM). BM are thin, specialized extracellular matrices found in all metazoans that are closely apposed to cell surfaces. They are important for providing mechanical stability to tissues and also provide signals that influence cellular polarity and guide cell and axon migrations. The major BM molecules identified in vertebrates have well conserved orthologs in C. elegans (Hutter et al., 2000). However, there are generally larger gene families for each molecule in vertebrates. For example, there are six type IV collagen genes in vertebrates vs. two in $C$. elegans and eleven laminin chain genes in vertebrates vs. four in $C$. elegans. The major $C$. elegans basement membrane proteins and their receptors are listed in Tables 1 and 2.

Table 1. Basement membrane molecules

\begin{tabular}{|c|c|c|c|c|}
\hline Common name & Gene & Isoforms $^{\mathbf{a}}$ & LOF phenotype(s) & References \\
\hline Collagen IV $\alpha 1$ & $e m b-9$ & 1 & $\operatorname{Emb}(2-3 X)$ & Guo et al., 1991; Gupta et al, 1997 \\
\hline Collagen IV $\alpha 2$ & let-2 & 2 & $\operatorname{Emb}(2-3 \mathrm{X})$ & Sibley et al., 1993, 1994 \\
\hline Collagen XVIII & cle-1 & 4 & Neuro, gonad morph & Ackley et al., 2001, 2003 \\
\hline Fibulin-1 & $f b l-1$ & 2 & Gonad morph & $\begin{array}{l}\text { Hesselson et al., 2004; Kubota et al., } \\
2004\end{array}$ \\
\hline Hemicentin & him-4 & 2 & $\begin{array}{l}\text { Tissue adhesion, } \\
\text { aneuploidy }\end{array}$ & Vogel and Hedgecock, 2001 \\
\hline Laminin $\alpha A$ & lam-3 & 1 & Emb, Acc & Huang et al., 2003 \\
\hline Laminin $\alpha B$ & epi-1 & 2 & Emb, Acc, Ste & Huang et al., 2003 \\
\hline Laminin $\beta$ & lam-1 & 1 & N.D. & \\
\hline $\operatorname{Laminin} \gamma$ & lam-2 & 1 & N.D. & \\
\hline Nidogen & nid-1 & 3 & Neuro & $\begin{array}{c}\text { Kang and Kramer, 2000; Kim and } \\
\text { Wadsworth, } 2000\end{array}$ \\
\hline Osteonectin/SPARC & ost-1 & 1 & Acc (L1-L2) & Fitzgerald and Schwarzbauer, 1998 \\
\hline Papilin & ppn-1 & 3 & Emb (hyp enclosure) & Kramerova et al., 2000 \\
\hline Perlecan & unc-52 & $>5$ & Pat & Rogalski et al., 1993, 1995 \\
\hline
\end{tabular}

In $C$. elegans, BMs surround all internal tissue surfaces, covering body wall muscles, pharynx, intestine, gonad and the pseudocoelomic face of the hypodermis. The BM covering the ventral and dorsal nerve cords interposes between motor neurons and muscles and forms part of the neuromuscular junctions, as is also the case in vertebrates. The thickness of the BM can differ between tissues (Figure 1), ranging from 20-100 nm (Huang et al., 2003). Temporal and/or tissue-specific differences in expression or localization of several BM components have been described. These differences result in different molecular compositions of BMs. For example, type collagen IV is not in all BMs, the two forms of laminin are differentially distributed, and perlecan is only present in BMs associated with muscle cells. 
Table 2. Receptors for basement membrane molecules

\begin{tabular}{|c|c|c|c|c|}
\hline Common name & Gene & Isoforms $^{\text {a }}$ & LOF phenotype $(s)^{b}$ & References \\
\hline Dystroglycan & dgn-1 & 1 & Ste, Neuro & $\begin{array}{c}\text { R. Johnson, S. Kang and J. Kramer, } \\
\text { unpublished results }\end{array}$ \\
\hline Dystroglycan & $d g n-2$ & 1 & N.D. & \\
\hline Dystroglycan & dgn-3 & 1 & N.D. & \\
\hline Glypican & gpn-1 & 1 & N.D. & \\
\hline Glypican & lon-2 & 1 & Lon & \\
\hline Integrin $\alpha$ & ina-1 & 1 & Acc (L1) & Baum and Garriga, 1997 \\
\hline Integrin $\alpha$ & pat-2 & 1 & Pat & Williams and Waterston, 1994 \\
\hline Integrin $\beta$ & pat-3 & 1 & Pat & Williams and Waterston, 1994 \\
\hline LAR-RPTP & $p t p-3$ & 3 & Emb (low penetrance) & Harrington et al., 2002 \\
\hline Syndecan & $s d n-1$ & 2 & Egl & Minniti et al., 2004 \\
\hline
\end{tabular}

${ }^{\text {a }}$ Protein isoforms resulting from alternative splicing and/or alternative promote usage.

${ }^{\mathrm{b}}$ Only the earliest or most prominent phenotype(s) are indicated. Abbreviations: Acc, larval arrest; Egl, egg-laying defective; Emb, embryonic lethal; Lon, long; Neuro, nervous system defects; Pat, paralyzed at two-fold; Ste, sterile.

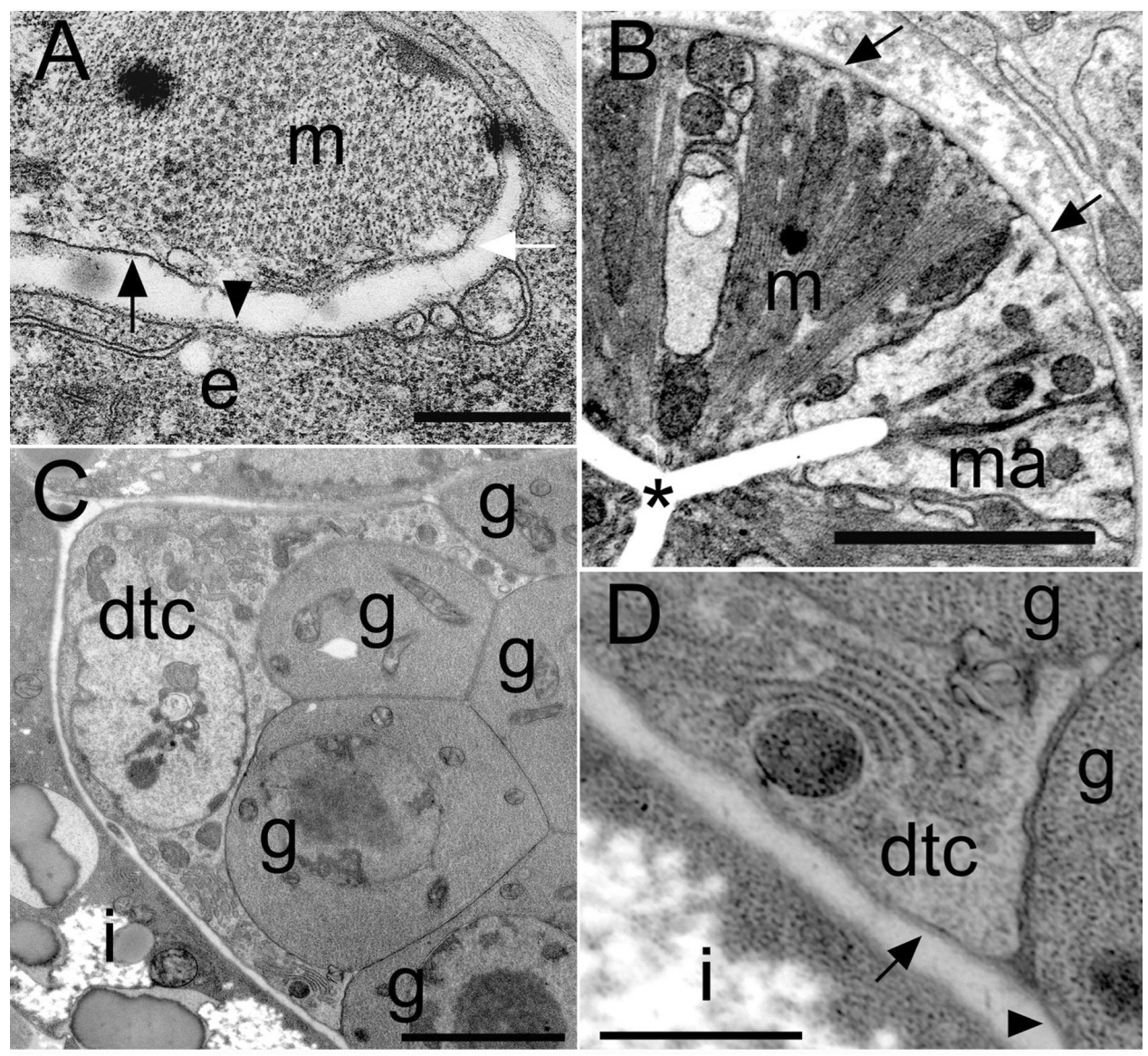

Figure 1. C. elegans basement membrane morphologies. (A) Body wall muscle (m) and epidermis (e) have thin sheets of BM. The epidermal BM has a lollipop-like appearance (arrowhead). The outward-facing body wall muscle BM (white arrow) is thicker than the inward-facing BM (black arrow). (B) The BM surrounding the pharynx is very thick (arrows). (C) The distal tip cell (dtc) extends processes to cover part of the germ cells (g). The gonad and intestine (i) are covered by separate BMs. (D) The dtc has a thick BM (arrow), whereas a thinner BM (arrowhead) covers the germ cells. Scale bars: $5 \mu \mathrm{m}$ in A-C; $1 \mu \mathrm{m}$ in D. Reprinted from Huang et al. (2003), with permission of The Company of Biologists Ltd. 
For all BM molecules that have been appropriately studied, multiple interactions with other BM molecules have been identified (Figure 2). The in vivo validity of many of these interactions remains to be established, but the numerous potential interactions between a large number of molecules predicts great diversity in composition and structure of BMs.

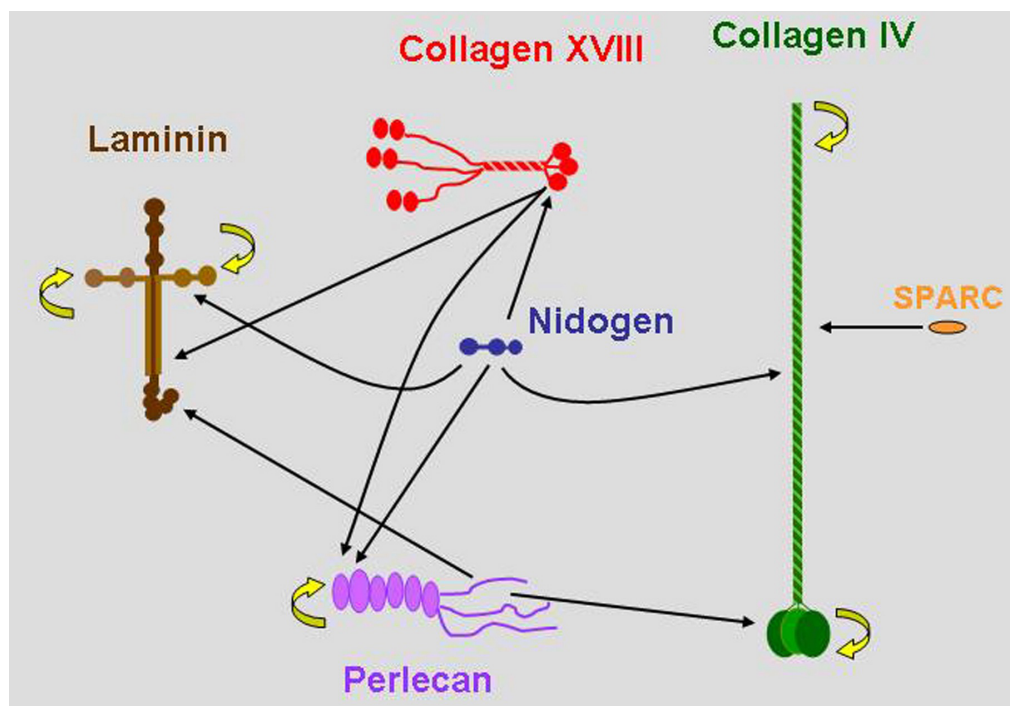

Figure 2. C. elegans type IV collagen genes. Major basement membrane molecules are represented in approximately appropriate relative sizes. Arrows represent biochemically defined interactions, mostly derived from studies of vertebrate molecules. The abilities of collagen IV and laminin to polymerize and perlecan to oligomerize are indicated by curved yellow arrows.

\section{Collagen type IV}

Type IV collagen is a heterotrimeric BM molecule containing two $\alpha 1$-like and one $\alpha 2$-like chain. Vertebrates have three $\alpha 1$ and three $\alpha 2$-like genes, while $C$. elegans has one of each, emb-9 and let-2 respectively. The LET-2 $\alpha 2$ chain is produced in two isoforms via alternative splicing that dramatically switches at the end of embryogenesis, such that one form predominates in embryos and the other in larvae and adults (Sibley et al.,1993). Interestingly, vertebrate and Drosophila $\alpha 1 / \alpha 2$ genes are found as divergently transcribed pairs with overlapping promoters, while the genes have separated onto different chromosomes in C. elegans (Guo and Kramer, 1989). Collagen IV has a short non-helical amino-terminal domain, a long Gly-X-Y repeat domain with numerous small interruptions and a highly conserved carboxyl-terminal globular NC1 domain. It polymerizes into a disulfide-bonded polygonal network via tetramerization between amino-terminal domains and dimerization between NC1 domains.

Most emb-9 and let-2 mutations are missense alterations of Gly residues in the Gly-X-Y repeat domains (Figure 3; Guo et al., 1991; Gupta et al., 1997; Sibley et al., 1994). Such alterations are expected to inhibit folding/stability of the triple-helical structure and have been shown to inhibit secretion of the mutant collagen. However, alterations of different Gly residues result in phenotypes of differing severities. Gly substitution mutations are generally highly temperature-sensitive, with greater intracellular accumulation and more severe phenotypes occurring at higher temperatures (Figure 4; Gupta et al. 1997). The most severe phenotype is arrest at about the two-fold stage of embryogenesis due to muscle detachment from the body wall resulting from the forces of muscle contraction. Null mutants actually elongate further, to the three-fold stage, indicating that the missense proteins interfere with the function of other molecules (Gupta et al., 1997).

Although type IV collagen had been considered a ubiquitous component of basement membranes, in $C$. elegans it is absent from the regions of the hypodermis between muscle quadrants and from the pseudocoelomic face of muscle cells (Graham et al., 1997). Type IV collagen is found in BMs on the pharynx and intestine, but is not synthesized in these tissues. Epitope-tagged $\alpha 1$ (IV) collagen expressed in body wall muscles was shown to assemble into these BMs, indicating some means of promoting its assembly specifically on these tissues. These studies show that type IV collagen secreted into the pseudocoelomic space can selectively assemble on appropriate tissue surfaces. 
Many $\mathrm{Y}$ position lysine residues in the Gly-X-Y repeats of type IV collagen are hydroxylated by lysyl hydroxylase and subsequently glycosylated within the ER/Golgi. Mutations in the single C. elegans lysyl hydroxylase gene, let-268, block secretion of collagen IV and result in a phenotype similar to that of mutations in emb-9 or let-2 (Norman and Moerman, 2000), demonstrating that this modification is essential for collagen IV function.

The phenotype of a mouse knockout of the $\alpha 1 / \alpha 2$ (IV) gene pair (Poschl et al., 2004) supports the conclusions of the C. elegans' studies of collagen IV mutants. Organogenesis proceeds normally and BM's can form but they cannot withstand normal mechanical stresses in the absence of type IV collagen.

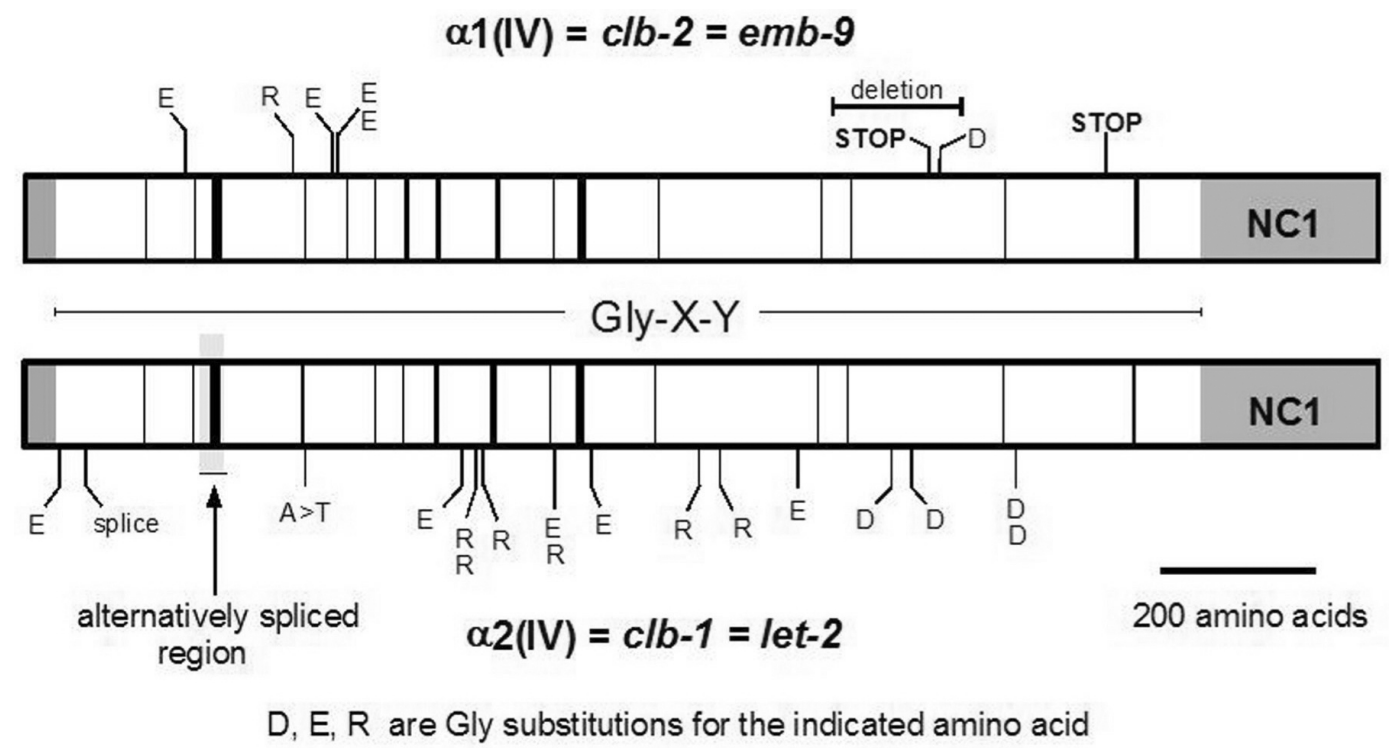

Figure 3. . elegans type IV collagen genes. The a1 and a2(IV) collagen chains are represented with amino and carboxyl-terminal domains grayed. The central Gly-X-Y domain is open with black bars representing interruptions of the repeats. Locations of known mutations are indicated and the alternatively spliced region of LET-2 is highlighted.

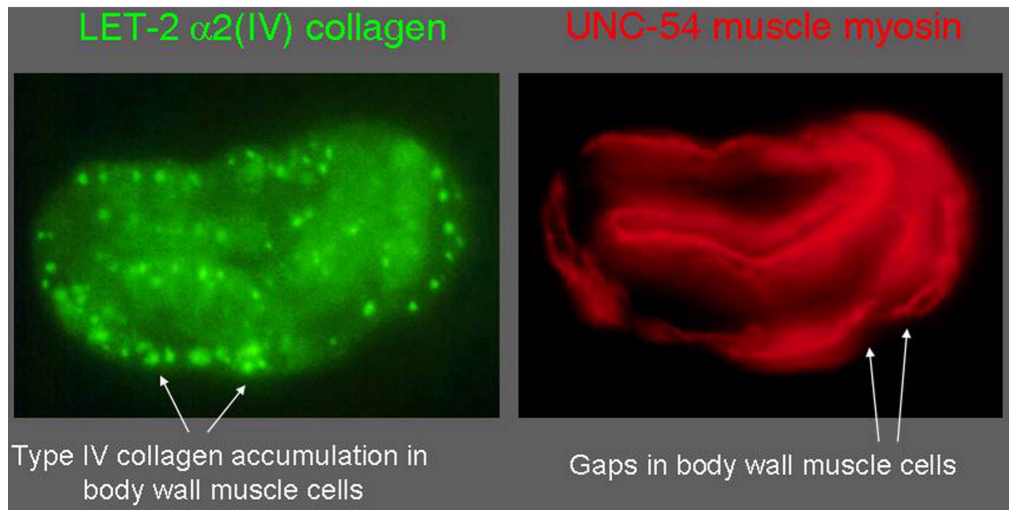

Figure 4. Intracellular accumulation of mutant type IV collagen. In a collagen IV $\alpha 1$ chain missense mutant, emb-9( $g 23)$, the LET-2 $\alpha 2$ chain accumulates within body wall muscle cells and is not secreted. EMB-9 also fails to be secreted. In these mutants muscle cells detach from the body wall, resulting in gaps in the body wall muscle quadrants.

\section{Laminins}

Laminins are large heterotrimeric glycoproteins comprised of an $\alpha, \beta$ and $\gamma$ chain. C. elegans has two $\alpha$ ( $\alpha \mathrm{A}$ LAM-3 and $\alpha$ B EPI-1) and single $\beta$ (LAM-1) and $\gamma$ (LAM-2) chains, resulting in two laminin isoforms (Figure 5). In contrast, vertebrates have multiple $\alpha, \beta$ and $\gamma$ chains, resulting in more than 15 laminin isoforms. The vertebrate 
family of netrins and UNC-6 in C. elegans are structurally related to $\beta$-laminin, but are not generally considered part of the laminin family.

The two $C$. elegans laminin forms have overlapping but distinct localization patterns. Both forms appear between germ layers near the end of gastrulation, with $\beta \mathrm{A}$ more concentrated around pharyngeal and intestinal precursors and $\alpha \mathrm{B}$ more concentrated on myoblasts and epidermis (Huang et al., 2003). In larval and adult animals $\alpha \mathrm{A}$ is found on the pharynx, intestine and excretory cell, but is more concentrated on the spermatheca, nerve ring and nerve cords. In contrast, $\alpha \mathrm{B}$ is found on pharynx, intestine, body wall muscle and hypodermis, but is concentrated over the gonad, accessory muscles and coelomocytes. Laminin $\alpha \mathrm{A}$ can localize to mispositioned axons, suggesting that there is a receptor for this laminin form on at least some axons.
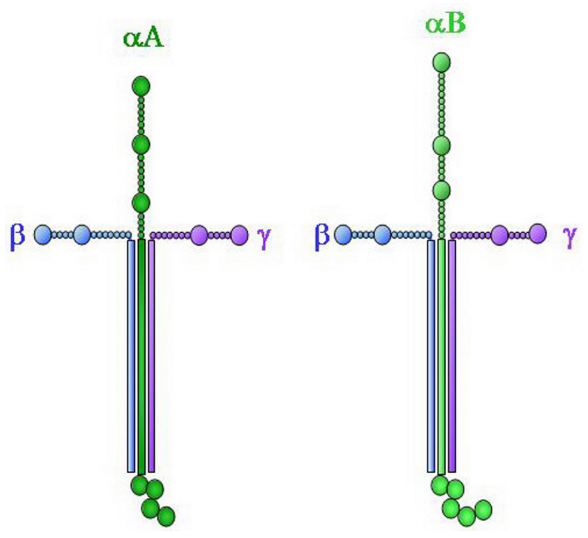

\begin{tabular}{|ccc|} 
Chain & Gene & $\begin{array}{c}\text { Vertebrate } \\
\text { equivalent }\end{array}$ \\
\hline$\alpha \mathrm{A}$ & lam-3 & $\alpha 1 / \alpha 2$-like \\
$\alpha \mathrm{B}$ & epi-1 & $\alpha 3 / \alpha 5$-like \\
$\beta$ & lam-1 & \\
$\gamma$ & lam-2 & \\
\hline
\end{tabular}

Figure 5. Structures of the two predicted C. elegans laminin molecules. The two structures differ only by which $\alpha$ chain is present.

The putative null mutation, epi-1(rh199) (Huang et al., 2003; Zhu et al., 1999), results in 27\% of animals developing to sterile adults, while the remainder arrest as embryos or larvae. In contrast, all lam-3 (n2561) animals arrest as embryos $(51 \%)$ or L1 stage larvae (49\%). Double RNAi against lam-3 and epi-1 results in a more severe phenotype, $85 \%$ embryonic and $15 \%$ L1 stage arrest, indicating that the two laminin forms have some independent essential functions.

In lam-3 animals the pharyngeal BM is defective and cells extend away from the pharynx, adhering to adjacent muscle or epidermal cells (Huang et al., 2003). Other tissues are relatively normal, consistent with the particularly strong concentration of LAM-3 on the pharynx. In epi-1 mutants multiple tissues, excluding the pharynx, have BM structural defects and failures of cellular polarization, adhesion, and organization. The defects in $\mathrm{BM}$ structure indicate that laminin is critical for normal BM assembly and/or stability and the range of phenotypes demonstrate roles for laminin in numerous developmental processes.

Several epi-1 alleles were generated in a screen for CAN cell migration defects (Forrester and Garriga, 1997; Forrester et al., 1998). These epi-1 mutants also have defects in migration of other cells and the outgrowth of some axons. The mutants display Unc, Egl and Muv phenotypes, but are generally less severe than the mutants described above. These results indicate that cell migration functions of laminin can be disrupted without causing severe disruption of laminin or BM structure.

\section{Perlecan}

The C. elegans ortholog of the ubiquitous vertebrate BM heparan sulfate proteoglycan perlecan is encoded by unc-52 (Rogalski et al., 1993). UNC-52 is only detected in BMs associated with muscle cells (Figure 6). Under body wall muscles it is concentrated beneath the muscle dense bodies and M-lines (Francis and Waterston, 1991; Mullen et al., 1999; Rogalski et al., 1993). A large number of UNC-52 isoforms can be generated by alternative splicing. Three major forms, termed short(S), medium(M) and long(L), differ by presence or absence of domains IV and/or V at the carboxyl end of the molecule. The $\mathrm{M}$ form is sufficient for normal muscle function and the significance of the other forms is unclear. Additionally exons 16-19 of domain IV can be alternatively spliced (Figure 7; Mullen et al., 1999; Rogalski et al., 1995; Rogalski et al., 1993). 
Strong unc-52 alleles are likely to eliminate or severely reduce unc-52 function and they cause the Pat phenotype. Body wall muscledense bodies fail to form and sarcomeres are not properly organized, resulting in arrest at the two-fold stage of embryogenesis (Hresko et al., 1994; Rogalski et al., 1995; Williams and Waterston, 1994). Viable, paralyzed $u n c-52$ alleles all affect the alternatively spliced exons 16-18 of domain IV (Rogalski et al., 1995). These mutations are expected to only affect a subset of UNC-52 isoforms. They also have somatic gonad defects, possibly due to defects in sheath or uterine myoepithelioid cells. Thirteen intragenic revertants of these viable Unc alleles were all shown to alter the splice acceptors of the exons affected in the original mutant, suggesting that exon skipping can ameliorate the Unc phenotype.
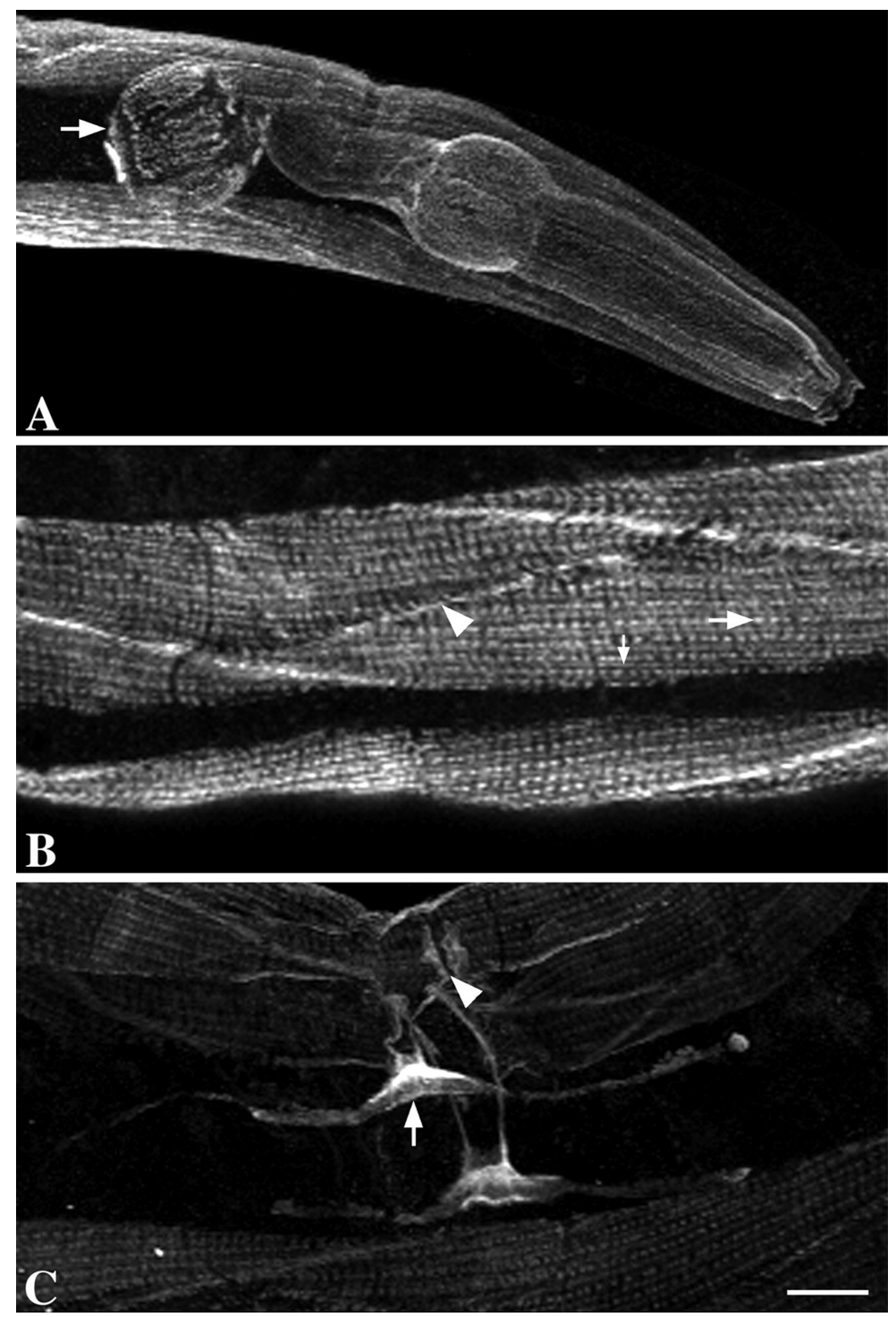

Figure 6. Immunolocalization of UNC-52 in adult hermaphrodites. Wild-type hermaphrodites were labeled with GM1. (A) shows the head from a young adult. The arrow indicates the terminal bulb of the pharynx. Note the punctate pattern over this region. (B) shows a section of the body-wall muscles from a young adult. The large arrow indicates a dense body, the small arrow indicates an M-line, and the arrowhead indicates the margin of a body-wall muscle cell. (C) shows the uterine region from an older adult (dorsal view). The arrow indicates the base of the uterine muscles, whereas the arrowhead indicates the vulva. Bar, $10 \mu \mathrm{m}$. Reprinted from Molecular Biology of the Cell (Mol. Biol. Cell 1999 10: 3205-3221) with the permission of The American Society for Cell Biology.

sup-38 mutations can suppress the muscle structural defects of viable unc-52 mutants, but not the defects of gonad structure (Gilchrist and Moerman, 1992). Putative sup-38 null alleles cause maternal-effect lethality but no apparent defect on muscle structure. The identity of SUP-38 is currently unknown. 
mec-8 mutations cause synthetic lethality in combination with unc-52 viable alleles (Lundquist and Herman, 1994). MEC-8 is an RNA binding protein that can regulate alternative splicing of unc-52 (Lundquist et al., 1996). Lethality presumably occurs because splice variants lacking the mutant exon are not produced. MEC-8 is a nuclear protein found in hypodermis, not muscle, and over-expression only in hypodermis can suppress unc-52 phenotypes (Spike et al., 2002). The synthetic lethality of mec-8; unc-52 mutants is only rescued by expression of MEC-8 in hypodermis. Finally, mosaic analyses showed that $u n c-52(+)$ is not required in muscle cells and are consistent with its requirement in hypodermal cells. These results indicate that UNC-52 is not synthesized by muscle cells, as previously thought (Moerman et al., 1996; Mullen et al., 1999), but is synthesized and secreted by the hypodermis. The fact that laser ablation of body wall muscle cells causes gaps in the normally continuous localization of UNC-52 (Moerman et al., 1996) shows that the localization of UNC-52 is specifically directed by muscle cells.

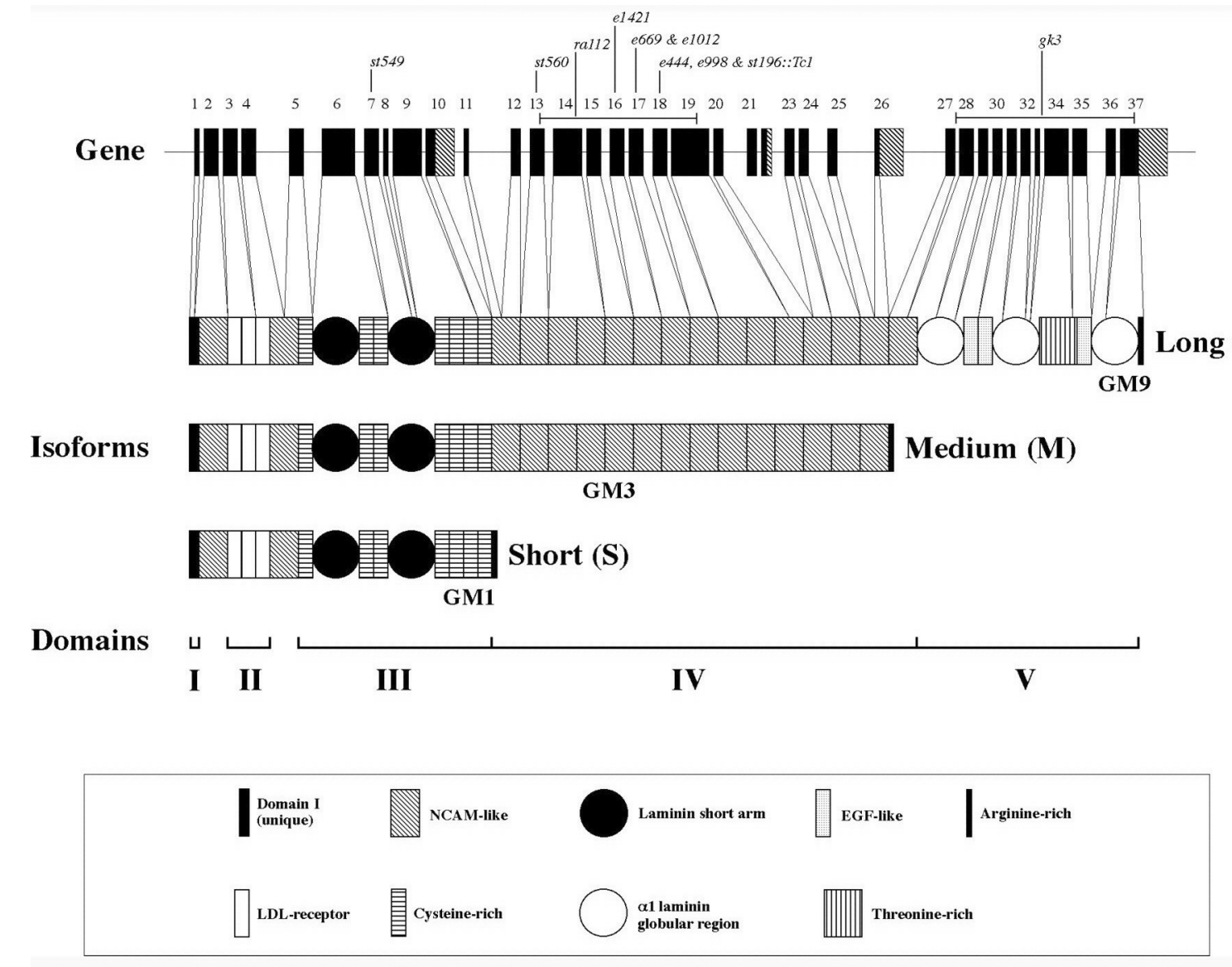

Figure 7. Structure of the $u n c-52$ gene and protein products. The $u n c-52$ gene consists of 37 exons and spans over $20 \mathrm{~kb}$. Exons (boxes), introns (lines), and the three classes of protein products are shown. Mutant alleles used in this study are also indicated. The longest ORF encodes a protein of 3375 amino acids that is homologous to the mammalian heparan sulfate basement membrane proteoglycan perlecan. Like mammalian perlecan, this polypeptide can be divided into five domains (I-V). The first domain is unique, whereas the remaining four domains show similarity to the low-density lipoprotein receptor (domain II), $\alpha$-laminin (domains III and V), and NCAM (domain IV). Additional isoforms are generated through alternative splicing of exons encoding alternative $\mathrm{C}$ termini, indicated on the gene as shaded regions. The various protein modules are indicated with shaded boxes or circles. Reprinted from Molecular Biology of the Cell (Mol. Biol. Cell 1999 10: 3205-3221) with the permission of The American Society for Cell Biology.

Viable unc-52 alleles enhance the distal tip cell migration defects that result from mutations in the netrin/UNC-6 guidance system (Merz et al., 2003). unc-52 double mutants with unc-6, unc-40 or unc-5 all have increased frequency of distal tip cell migration defects. RNAi specific to the major UNC-52 size forms indicate that the $\mathrm{M}$ form is important for affecting distal tip cell migration. Loss of function mutants of two TGF- $\beta$-like genes, $d b l-1$ and $u n c-129$, enhance $u n c-5$ partial loss of function mutations. The unc-52 enhancement of unc-5 distal tip cell migration defects is suppressed by reduction of function mutations in the growth factor genes. These results suggest that viable unc-52 mutations effectively increase growth factor signaling, possibly due to failure to sequester/localize the growth factors properly. Evidence for growth factor binding to perlecan and modulation of signaling has been demonstrated in vertebrates (Jiang and Couchman, 2003. 


\section{Nidogen}

Nidogen/entactin is a ubiquitous BM glycoprotein that consists of two amino (G1, G2) and one carboxyl (G3) globular domains that are connected by a rod domain composed primarily of EGF repeats. There are two closely related nidogen genes in mammals, but only a single $C$. elegans gene, nid-1. Three alternative splice variants of nid-1 alter the number of EGF repeats in the rod domain (Figure 8; Kang and Kramer, 2000). Vertebrate biochemical studies show that the G3 domain binds with high affinity to the laminin $\gamma$ chain, while the G2 domain can bind perlecan and type IV collagen. The ability of nidogen to form a ternary complex with laminin and type IV collagen led to the suggestion that it may function as a linker between BM proteins.

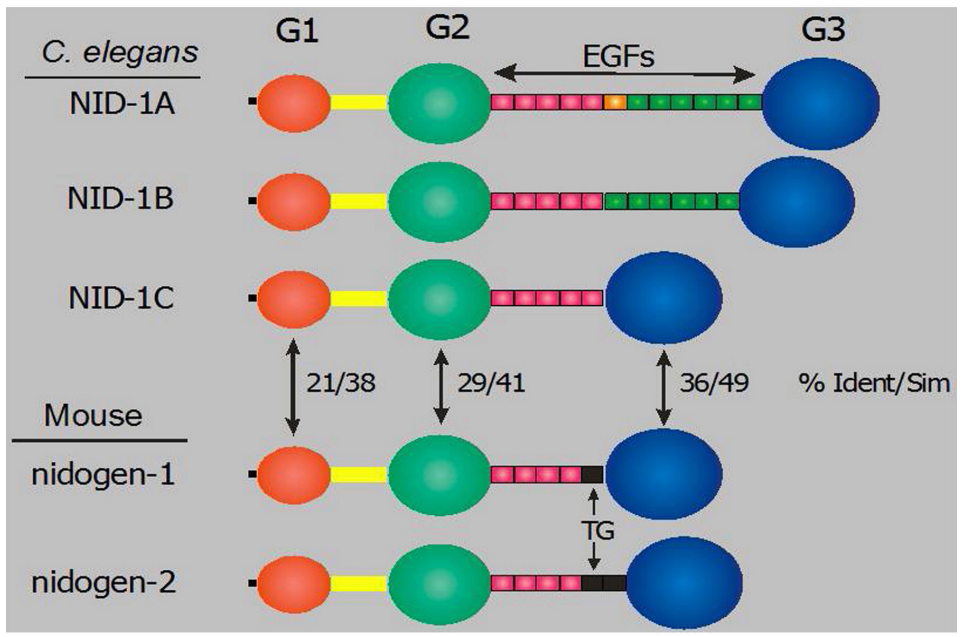

Figure 8. Structures of $\boldsymbol{C}$. elegans and mouse nidogens. The domain structures of the three NID-1 alternative splice isoforms and two mouse nidogen genes are illustrated. The percentages of amino acid sequence identity/similarity between $C$. elegans and globular domains are shown. Thyroglobulin-like modules in the mouse proteins are indicated by TG.

In C. elegans, NID-1 is localized in all BMs but is highly concentrated on the nerve ring, sublateral nerve cords, spermatheca and distal tip cells (Figure 9; Kang and Kramer, 2000). Three nid-1 alleles have been generated: cg119, a molecular null; ur41, a nonsense allele; $c g 118$, an internal deletion that produces stable protein lacking the G2 domain (Kang and Kramer, 2000; Kim and Wadsworth, 2000). The ur41 allele is not null, as it produces a stable G1/G2 domain fragment that is mislocalized (S. Kang and J. Kramer, unpublished results). Each of these mutants is viable and fertile, and have no obvious defect in localization of other BM proteins, demonstrating that nidogen is not essential for BM assembly.
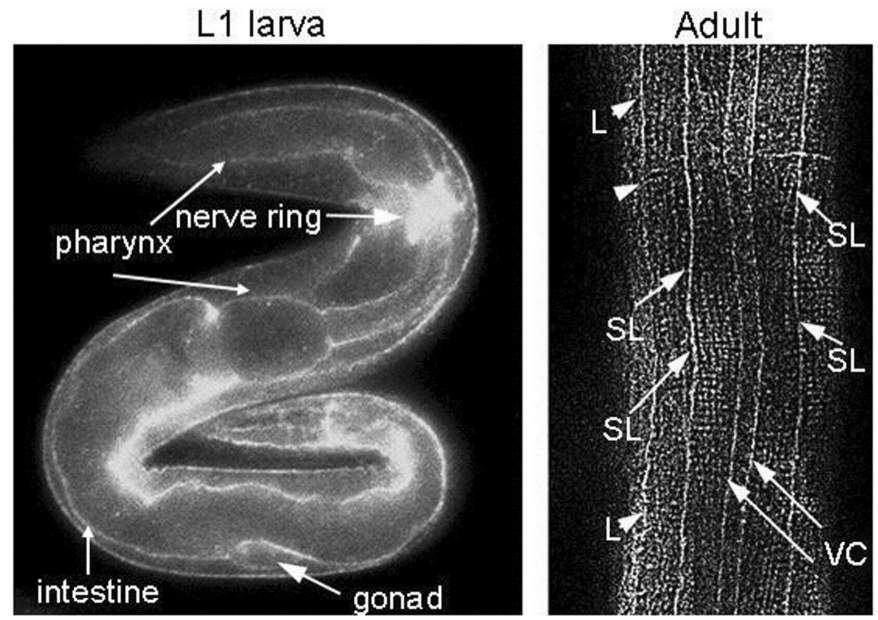

Figure 9. Immunolocalization of NID-1. An L1 larva and adult stained with anti-NID-1 G3 domain antibody. In the L1 animal staining of multiple basement membranes is seen, but NID-1 is most strongly concentrated around the nerve ring. In the adult animal, strong staining of sublateral nerve cords (SL), the ventral nerve cord/muscle edge (VC) and lateral edge of muscle quadrants (L) is seen. 
The ur41 nonsense allele alters the dorso-ventral positioning of several axons (Kim and Wadsworth, 2000). For example, the dorsal sublateral cords are frequently positioned closer than normal to the dorsal midline and too many axons enter the right vs. left fascicle of the ventral cord. The affected axons do reach their normal targets, but these results show that NID-1 influences neural guidance decisions. NID-1 also affects synaptic organization and function (Ackley et al., 2003). NID-1 is closely associated, but not precisely colocalized, with synaptic markers. However, both pre- and post-synaptic markers show that nid-1 synapses are significantly less well compacted than in wild-type animals, appearing smeared along the nerve cord. These mutants are substantially resistant to a cholinergic agonist, indicating a transmission deficit. They also display uncoordinated behaviors in thrashing assays. All of these defects are more penetrant for the $\operatorname{cg} 119$ null allele than for the $\operatorname{cg} 118$ internal deletion, indicating that NID-1 lacking the G2 domain retains a high degree of normal function.

\section{Collagen type XVIII}

The closely related vertebrate BM collagen type XV and type XVIII genes have a single ortholog in $C$. elegans, cle-1 (Figure 10). The carboxyl-terminal domain of collagen XVIII, termed endostatin (ES), has been characterized as having potent anti-angiogenic activity. This domain is the most highly conserved between the vertebrate and $C$. elegans proteins. cle-1 generates at least three protein isoforms by utilizing three promoters and alternative splicing (Figure 11; Ackley et al., 2001). The two longer CLE-1 isoforms are synthesized by neurons, an unusual situation for any collagen. CLE-1 is broadly distributed at low levels throughout BMs, but is notably concentrated on the nervous system. Mutations that truncate the molecule, removing the C-terminal domain, result in altered migrations of multiple neurons and the distal tip cells, and also cause ray fusions in the male tail. Defects in the extension of PLM and DA/DB axons were also observed. Loss of CLE-1 alters the extents of cell migrations, but does not generally alter their guidance.

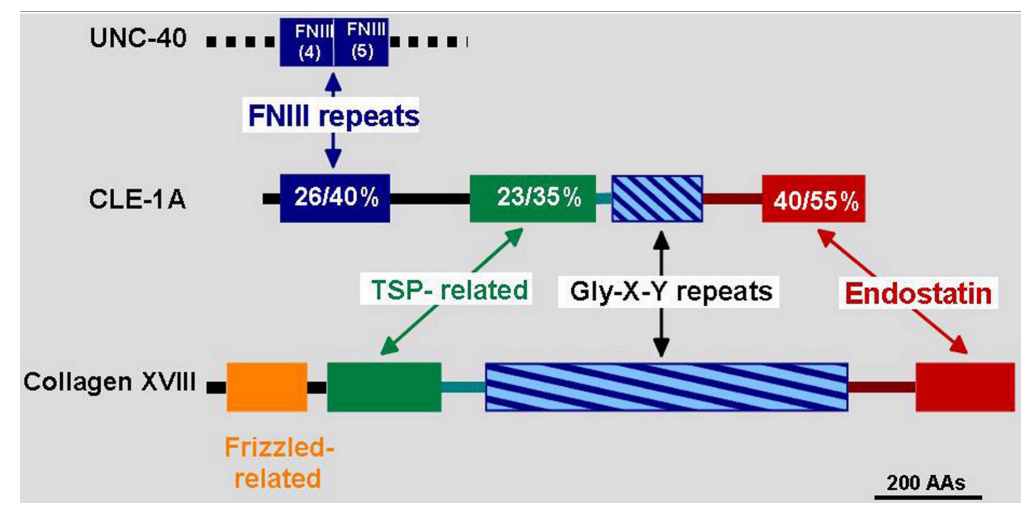

Figure 10. Comparison of CLE-1 and mouse collagen XVIII structures. The domains of CLE-1 and collagen XVIII are illustrated with percentages of amino acid sequence identity/similarity indicated in the CLE-1 representation. The longest form CLE-1A is shown. The amino terminus of CLE-1A is related to UNC-40, while mouse protein is related to Frizzled. TSP-related indicates similarity to the thrombospondin amino terminal domain.

A region immediately carboxyl-terminal to the Gly-X-Y domain of collagen XVIII, the association domain (AD), has been shown to promote homo-trimerization of the carboxyl-terminal ES domain in vertebrates and $C$. elegans (Ackley et al., 2001). Transgenes expressing the complete AD plus ES domain are able to rescue ALM/AVM migration defects of cle-1 truncation mutants. However, expression of the ES domain alone does not rescue and in fact causes migration defects in the wild-type background. These results suggest that trimeric collagen XVIII may promote migration, while monomeric ES domains may act to inhibit migrations. Similar results have been observed in studies of vertebrate cultured cells (Kuo et al., 2001). 


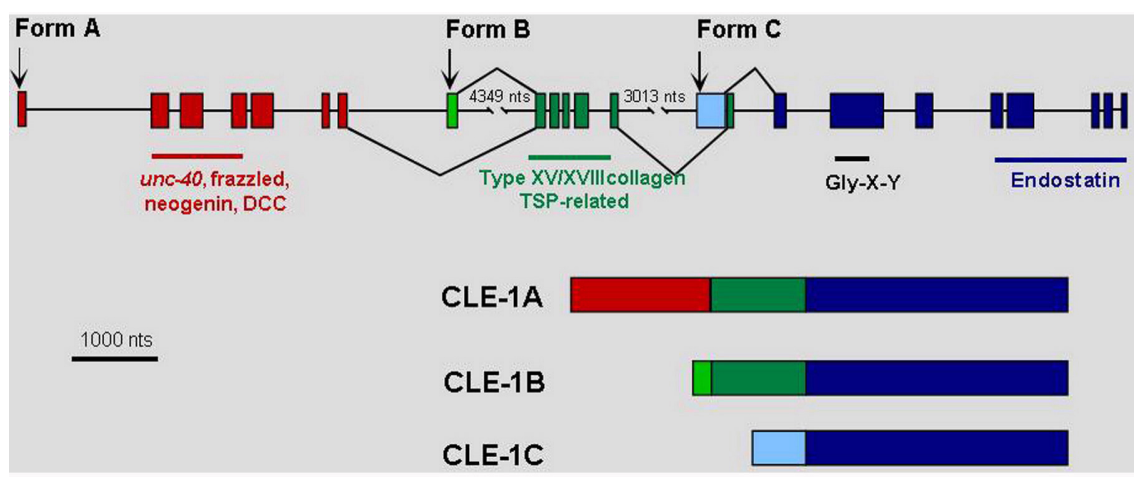

Figure 11. Structure of the cle-1 and protein isoforms. The starts of the three CLE-1 forms, which are transcribed from separate promoters, are shown by arrows above the gene structure. Alternative splicing is indicated by lines above and below the structure connecting between exons. The resulting protein products are illustrated below the gene structure.

CLE-1 is concentrated on the nervous system, does not specifically colocalize with synaptic markers, but does affect synaptic organization and function (Ackley et al., 2003). Both pre- and post-synaptic markers show that cle-1 mutants have enlarged, more widely spaced synapses relative to wild-type animals. The mutants display uncoordinated behaviors in thrashing assays and have altered responses to a cholinergic agonist and an acetylcholinesterase inhibitor.

\section{SPARC/Osteonectin}

The C. elegans ortholog of the BM associated protein SPARC/osteonectin/BM40 is encoded by ost-1 (Schwarzbauer and Spencer, 1993). In vertebrate studies SPARC has been shown to modulate cell matrix interactions, inhibit proliferation and affect growth factor signaling. Recombinant OST-1 amino- and carboxyl-terminal domains bind calcium, without inducing the significant conformational change seen in the vertebrate protein (Maurer et al., 1997; Schwarzbauer and Spencer, 1993). OST-1 was further shown to bind human type I and type IV collagens in a calcium-dependent manner with similar affinity as the human protein. These results suggest that OST-1 could interact with type IV collagen in C. elegans BMs.

ost-1 expression is restricted to body wall and sex muscles. High-copy transgenes carrying ost- 1 can cause Unc and protruding vulva phenotypes (Schwarzbauer and Spencer, 1993). This suggests that over expression of OST-1 is detrimental, although other detrimental effects of the high copy arrays were not ruled out. An OST-1::GFP fusion protein shows localization to body wall and vulval muscle, and on the surfaces of the pharynx and gonad (Fitzgerald and Schwarzbauer, 1998). RNAi against ost-1 resulted in a mix of Emb at the pre-comma stage and Acc at the L1 stage, with apparently normal morphology. Escaper adults were clear and small, and were also frequently Ste or Egl. The spectrum of phenotypes suggests that OST-1 has multiple functions throughout development.

\section{Fibulin}

Fibulins are widely expressed basement membrane proteins, encoded by a family of five genes in vertebrates, that all contain a number of calcium-binding EGF modules and a family-specific carboxyl-terminal domain. The single $C$. elegans gene, $f b l-1$, is most similar to vertebrate fibulin- 1 and, like it, encodes alternative splice forms with differing carboxyl-termini. Fibulin-1 can bind laminin and nidogen, as well as several other matrix molecules. Knock-out of mouse fibulin-1 results in ruptures of small blood vessels and perinatal lethality.

fbl- 1 mutations were identified as suppressors of gonad morphogenesis defects resulting from loss-of-function mutations in the matrix metalloproteinase genes gon-1 or mig-17 (Hesselson et al., 2004; Kubota et al., 2004). There is a complete failure of gonad arm extension in gon-1 mutants, while mig-17 mutants only display abnormal guidance of the distal tip cells. MIG-17 is an ADAM (a disintegrin and metalloproteinase) and GON-1 is an ADAMTS (ADAM plus thrombospondin repeats). ADAMs can cleave basement membrane proteins, but can also act as sheddases, proteolytically releasing the ectodomains of cell surface proteins.

$f b l-1$ loss-of-function mutations result in a grossly distended proximal gonad and failure of the centripetal migration of the distal tip cells, possibly due to physical hindrance by the proximal gonad. Double mutants of gon- 1 with fbl-1 loss-of-function show partial restoration of arm extension (Hesselson et al., 2004), suggesting that they 
may function antagonistically. In contrast, mig-17 mutations are suppressed by $f b l-1$ gain-of-function (gof) mutations, all three of which affect the third calcium-binding EGF module (Kubota et al., 2004). The FBL-1C, but not FBL-1D, isoform is required for mig-17 suppression and its accumulation in the gonad basement membrane is reduced in mig-17 mutants. The presence of FBL-1C(gof) must overcome the need for MIG-17, possibly by altering interaction with another protein that is normally the target of MIG-17.

\section{Hemicentin}

Hemicentin, HIM-4, is not strictly a BM component, but is a conserved extracellular matrix protein (Vogel and Hedgecock, 2001). It is a large protein with unique amino and carboxyl-terminal domains flanking $48 \mathrm{Ig}$ and 3 EGF modules, that is expressed in body wall muscles and gonadal leader cells (dtc and linker cell). GFP-hemicentin forms tracks between internal tissues and the basal surface of the hypodermis and accumulates around individual germ cells in the distal gonad. In him-4 mutants intestinal cells move abnormally relative to the body wall, ALM/PLM axons fail to induce hemidesmosome assembly in the overlying epidermis, male linker cell migration is abnormal, the uterus frequently prolapses, and there is a high degree of aneuploidy in the germ line. In all cases hemicentin appears to have an adhesive function. In the germline, hemicentin is thought to stabilize the partial cellularization of germ cells which may prevent kinetochore attachment by spindles from adjacent cells. A missense mutation in human hemicentin (also known as fibulin-6) has been associated with age related macular degeneration in one family.

\section{Integrins}

Integrins are transmembrane $\alpha / \beta$ heterodimers that can bind to many extracellular matrix molecules. Integrins are important for cell adhesion and migration, and can have roles in matrix assembly and remodeling. In vertebrates at least 24 distinct integrins are generated from $18 \alpha$ and $8 \beta$ chain genes. Only two integrins are predicted in $C$. elegans, derived from two $\alpha$, ina- 1 and pat-2, and a single $\beta$, pat-3, chain gene. INA-1 is most similar to laminin-binding integrins, while PAT-2 is similar to RGD-binding integrins.

PAT-3 is very broadly expressed, as expected for the sole integrin $\beta$ chain (Gettner et al., 1995). INA-1 is also broadly expressed in early embryos but by the completion of embryogenesis becomes restricted to neurons, migrating cells and cells in tissues undergoing morphogenesis (Baum and Garriga, 1997). The existence of the INA-1/PAT-3 heterodimer has been demonstrated by co-immunoprecipitation experiments (Baum and Garriga, 1997).

Both pat-3 and pat-2 mutants have the Pat (paralyzed at two-fold) phenotype and fail to form dense bodies or organize sarcomeres (Williams and Waterston, 1994). The similarity in phenotype to unc-52 perlecan mutants (Mullen et al., 1999; Rogalski et al., 1993) and the concentration of integrin at dense bodies (Francis and Waterston, 1991; Gettner et al., 1995) suggest that the PAT-2/PAT-3 integrin could bind UNC-52 in the BM.

Several ina- 1 alleles were isolated in screen for mispositioned neurons (Baum and Garriga, 1997). Strong alleles, one of which is a likely null, cause arrest at the L1 larval stage and have a notched-head phenotype. Weak alleles can develop to fertile adults but can have the notched head phenotype and defects in gonad and vulval morphology. Multiple neurons fail to complete migrations in ina-1 mutants and mosaic analysis shows that ina-1 functions cell autonomously in these migrating neurons. Interestingly, axon extension/guidance are not generally defective in ina- 1 animals but defasciculation is seen, suggesting defects in adhesion between axons.

In vertebrates, expression of $\beta$ tail constructs ( $\beta$ integrin transmembrane and cytoplasmic domain) can exert a dominant negative effect on integrin function. Expression of a PAT-3 $\beta$ tail construct in muscles and gonad results in Pat, Unc and Egl phenotypes (Lee et al., 2001). Defects in distal tip cell migration and ovulation were also seen. Alteration of conserved tyrosines in the cytoplasmic tail, that are phosphorylated in vertebrates, reduced full-length PAT-3 function and enhanced the disruptive effects of the $\beta$ tail construct, suggesting that they have integrin regulatory functions in C. elegans as well. 


\section{References}

Ackley, B.D., Crew, J.R., Elamaa, H., Pihlajaniemi, T., Kuo, C.J., and Kramer, J.M. (2001). The NC1/endostatin domain of Caenorhabditis elegans type XVIII collagen affects cell migration and axon guidance. J. Cell. Biol. 152, 1219-1232. Abstract Article

Ackley, B.D., Kang, S.H., Crew, J.R., Suh, C., Jin, Y., and Kramer, J.M. (2003). The basement membrane components nidogen and type XVIII collagen regulate organization of neuromuscular junctions in Caenorhabditis elegans. J. Neurosci. 23, 3577-3587. Abstract

Baum, P.D., and Garriga, G. (1997). Neuronal migrations and axon fasciculation are disrupted in ina-1 integrin mutants. Neuron 19, 51-62. Abstract Article

Fitzgerald, M.C., and Schwarzbauer, J.E. (1998). Importance of the basement membrane protein SPARC for viability and fertility in Caenorhabditis elegans. Curr. Biol. 8, 1285-1288. Abstract Article

Forrester, W.C., and Garriga, G. (1997). Genes necessary for C. elegans cell and growth cone migrations. Development 124, 1831-1843. Abstract

Forrester, W.C., Perens, E., Zallen, J.A., and Garriga, G. (1998). Identification of Caenorhabditis elegans genes required for neuronal differentiation and migration. Genetics 148, 151-166. Abstract

Francis, R., and Waterston, R.H. (1991). Muscle cell attachment in Caenorhabditis elegans. J. Cell. Biol. 114, 465-479. Abstract Article

Gettner, S.N., Kenyon, C., and Reichardt, L.F. (1995). Characterization of beta pat-3 heterodimers, a family of essential integrin receptors in C. elegans. J. Cell. Biol. 129, 1127-1141. Abstract Article

Gilchrist, E.J., and Moerman, D.G. (1992). Mutations in the sup-38 gene of Caenorhabditis elegans muscle-attachment defects in unc-52 mutants. Genetics 132, 431-442. Abstract

Graham, P.L., Johnson, J.J., Wang, S.R., Sibley, M.H., Gupta, M.C., and Kramer, J.M. (1997). Type IV collagen is detectable in most, but not all, basement membranes of Caenorhabditis elegans and assembles on tissues that do not express it. J. Cell. Biol. 137, 1171-1183. Abstract Article

Guo, X., Johnson, J.J., and Kramer, J.M. (1991). Embryonic lethality caused by mutations in basement membrane collagen of C. elegans. Nature 349, 707-709. Abstract Article

Guo, X., and Kramer, J.M. (1989). The two Caenorhabditis elegans basement membrane (typeIV) collagen genes are located on separate chromosomes. J. Biol. Chem. 264, 17575-17582. Abstract

Gupta, M.C., Graham, P.L., and Kramer, J.M. (1997). Characterization of alpha 1(IV) collagen mutations in Caenorhabditis elegans and the effects of alpha 1 and alpha 2(IV) mutations on type IV collagen distribution. J. Cell. Biol. 137, 1185-1196. Abstract Article

Harrington, R.J., Gutch, M.J., Hengartner, M.O., Tonks, N.K., and Chisholm,A.D. (2002). The C. elegans LAR-like receptor tyrosine phosphatase PTP-3 and the VAB-1 Eph receptor tyrosine kinase have partly redundant functions in morphogenesis. Development 129, 2141-2153.

Hesselson, D., Newman, C., Kim, K.W., and Kimble, J. (2004). GON-1 and fibulin have antagonistic roles in control of organ shape. Curr. Biol. 14, 2005-2010. Abstract Article

Hresko, M.C., Williams, B.D., and Waterston, R.H. (1994). Assembly of body wall muscle and muscle cell attachment structures in Caenorhabditis elegans. J. Cell. Biol. 124, 491-506. Abstract Article

Huang, C.-C., Hall, D.H., Hedgecock, E.M., Kao, G., Karantza, V., Vogel, B.E., Hutter, H., Chisholm, A.D., Yurchenco, P.D., and Wadsworth, W.G. (2003). Laminin \{alpha\} subunits and their role in C. elegans development. Development 130, 3343-3358. Abstract Article 
Hutter, H., Vogel, B.E., Plenefisch, J.D., Norris, C.R., Proenca, R.B., Spieth, J., Guo, C., Mastwal, S., Zhu, X., Scheel, J., et al. (2000). Conservation and novelty in the evolution of cell adhesion and extracellular matrix genes. Science 287, 989-994. Abstract Article

Jiang, X., and Couchman, J.R. (2003). Perlecan and tumor angiogenesis. J. Histochem. Cytochem. 51, $1393-1410$. Abstract

Kang, S.H., and Kramer, J.M. (2000). Nidogen is nonessential and not required for normal type IV collagen localization in Caenorhabditis elegans. Mol. Biol. Cell 11, 3911-3923. Abstract

Kim, S., and Wadsworth, W.G. (2000). Positioning of longitudinal nerves in C. elegans by nidogen. Science 288 , 150-154. Abstract Article

Kramerova, I.A., Kawaguchi, N., Fessler, L.I., Nelson, R.E., Chen, Y., Kramerov, A.A., Kusche-Gullberg, M., Kramer, J.M., Ackley, B.D., Sieron, A.L., et al. (2000). Papilin in development; a pericellular protein with a homology to the ADAMTS metalloproteinases. Development 127, 5475-5485.

Kubota, Y., Kuroki, R., and Nishiwaki, K. (2004). A fibulin-1 homolog interacts with an ADAM protease that controls cell migration in C. elegans. Curr. Biol. 14, 2011-2018. Abstract Article

Kuo, C.J., LaMontagne, K.R., Jr., Garcia-Cardena, G., Ackley, B.D., Kalman, D., Park, S., Christofferson, R., Kamihara, J., Ding, Y.-H., Lo, K.-M., et al. (2001). Oligomerization-dependent regulation of motility and morphogenesis by the collagen XVIII NC1/endostatin domain. J. Cell. Biol. 152, 1233-1246. Article

Lee, M., Cram, E.J., Shen, B., and Schwarzbauer, J.E. (2001). Roles for beta pat-3 integrins in development and function of Caenorhabditis elegans muscles and gonads. J. Biol. Chem. 276, 36404-36410. Abstract Article

Lundquist, E.A., and Herman, R.K. (1994). The mec-8 gene of Caenorhabditis elegans affects muscle and sensory neuron function and interacts with three other genes: unc-52, smu-1 and smu-2. Genetics 138, 83-101. Abstract

Lundquist, E.A., Herman, R.K., Rogalski, T.M., Mullen, G.P., Moerman, D.G., and Shaw, J.E. (1996). The mec-8 gene of $C$. elegans encodes a protein with two RNA recognition motifs and regulates alternative splicing of $u n c-52$ transcripts. Development 122, 1601-1610. Abstract

Maurer, P., Sasaki, T., Mann, K., Gohring, W., Schwarzbauer, J.E., and Timpl, R. (1997). Structural and functional characterization of the extracellular calcium-binding protein BM-40/secreted protein, acidic, rich in cysteine/osteonectin from the nematode Caenorhabditis elegans. Eur. J. Biochem. 248, 209-216. Abstract Article

Merz, D.C., Alves, G., Kawano, T., Zheng, H., and Culotti, J.G. (2003). UNC-52/Perlecan affects gonadal leader cell migrations in C. elegans hermaphrodites through alterations in growth factor signaling. Dev. Biol. 256, 174-187. Abstract Article

Minniti, A.N., Labarca, M., Hurtado, C., and Brandan, E. (2004). Caenorhabditis elegans syndecan (SDN-1) is required for normal egg laying and associates with the nervous system and the vulva. J. Cell Sci. 117, 5179-5190. Article

Moerman, D.G., Hutter, H., Mullen, G.P., and Schnabel, R. (1996). Cell autonomous expression of perlecan and plasticity of cell shape in embryonic muscle of Caenorhabditis elegans. Dev. Biol. 173, 228-242. Abstract Article

Mullen, G.P., Rogalski, T.M., Bush, J.A., Gorji, P.R., and Moerman, D.G. (1999). Complex patterns of alternative splicing mediate the spatial and temporal distribution of perlecan/UNC-52 in Caenorhabditis elegans. Mol. Biol. Cell 10,3205-3221. Abstract

Norman, K.R., and Moerman, D.G. (2000). The let-268 locus of Caenorhabditis elegans encodes a procollagen lysyl hydroxylase that is essential for type IV collagen secretion. Dev Biol 227, 690-705. Abstract Article

Poschl, E., Schlotzer-Schrehardt, U., Brachvogel, B., Saito, K., Ninomiya, Y., and Mayer, U. (2004). Collagen IV is essential for basement membrane stability but dispensable for initiation of its assembly during early development. Development 131, 1619-1628. Abstract Article 
Rogalski, T.M., Gilchrist, E.J., Mullen, G.P., and Moerman, D.G. (1995). Mutations in the unc-52 gene responsible for body wall muscle defects in adult Caenorhabditis elegans are located in alternatively spliced exons. Genetics 139, 159-169. Abstract

Rogalski, T.M., Williams, B.D., Mullen, G.P., and Moerman, D.G. (1993). Products of the unc-52 gene in Caenorhabditis elegans are homologous to the core protein of the mammalian basement membrane heparan sulfate proteoglycan. Genes Dev. 7, 1471-1484. Abstract

Schwarzbauer, J.E., and Spencer, C.S. (1993). The Caenorhabditis elegans homologue of the extracellular calcium binding protein SPARC/osteonectin affects nematode body morphology and mobility. Mol. Biol. Cell 4, 941-952. Abstract

Sibley, M.H., Graham, P.L., von Mende, N., and Kramer, J.M. (1994). Mutations in the alpha2(IV) basement membrane collagen gene of Caenorhabditis elegans produce phenotypes of differing severities. EMBO J. 13, 3278-3285. Abstract

Sibley, M.H., Johnson, J.J., Mello, C.C., and Kramer, J.M. (1993). Genetic identification, sequence, and alternative splicing of the Caenorhabditis elegans alpha2(IV) collagen gene. J. Cell. Biol. 123, 255-264. Abstract Article

Spike, C.A., Davies, A.G., Shaw, J.E., and Herman, R.K. (2002). MEC-8 regulates alternative splicing of unc-52 transcripts in C. elegans hypodermal cells. Development 129, 4999-5008. Abstract

Vogel, B.E., and Hedgecock, E.M. (2001). Hemicentin, a conserved extracellular member of the immunoglobulin superfamily, organizes epithelial and other cell attachments into oriented line-shaped junctions. Development 128 , 883-894. Abstract

Williams, B.D., and Waterston, R.H. (1994). Genes critical for muscle development and function in Caenorhabditis elegans identified through lethal mutations. J. Cell. Biol. 124, 475-490. Abstract Article

Zhu, X., Joh, K., Hedgecock, E.M., and Hori, K. (1999). Identification of epi-1 locus as a laminin alpha chain gene in the nematode Caenorhabditis elegans and characterization of epi-1 mutant alleles. DNA Seq. 10, $207-217$. Abstract

All WormBook content, except where otherwise noted, is licensed under a Creative Commons Attribution License. 